\title{
Impact of liver fat on the response of plasma triacylglycerol to diets high and low in extrinsic sugars
}

\author{
A. Ahmad ${ }^{1}$, C. Isherwood ${ }^{1}$, J. D. Bell ${ }^{2}$, E. L. Thomas ${ }^{2}$, G. Frost ${ }^{3}$, M. Umpleby ${ }^{1}$ and B. A. Griffin ${ }^{1}$ \\ ${ }^{1}$ Faculty of Health \& Medical Sciences, University of Surrey, Guildford, Surrey GU2 7XH, ${ }^{2}$ Robert Steiner, MR Unit, \\ MRC Clinical Sciences Centre, Imperial College, London W12 OHS and ${ }^{3}$ Department of Medicine, Imperial College, \\ London SW7 2AZ, UK
}

The dietary extrinsic sugars, sucrose and fructose, have been implicated in the formation of high risk abnormalities in plasma lipoproteins, known as an atherogenic lipoprotein phenotype, primarily by elevating plasma triacylglycerol (TAG). The extent to which these sugars can elevate plasma TAG and alter lipoproteins may depend on their ability to promote the accumulation of fat and insulin resistance in the liver ${ }^{(1)}$. The interrelationship between dietary sugar, plasma TAG and percent liver fat was examined in a dietary intervention study in which plasma TAG was measured before and after two, 12 week diets that were high and low in extrinsic sugars. The diets were delivered in a randomised cross-over design to male participants $(n=25)$, aged 40-65 years, who demonstrated increased cardio-metabolic risk according to the previously described 'RISCK' study criteria ${ }^{(2)}$. The dietary model was based on NDNS data from an average intake of men aged 35-49 and 50-64 years ${ }^{(3)}$. It exchanged two-thirds of dietary carbohydrate with foods high and low in extrinsic sugars, and achieved target ratios of starch to sugar of 1:1.2 (High total sugar) and 3:1 (Low total sugar). Liver fat was measured at the start of the study by magnetic resonance spectroscopy (MRS). After the normalisation and adjustment of data for baseline values, there was a significant difference in plasma TAG following the high and low sugar diets $(P=0.003$, ANCOVA). The Figure shows the differential response of plasma TAG (geometric means) to the high and low sugar diets across quintiles of increasing percentage liver fat.

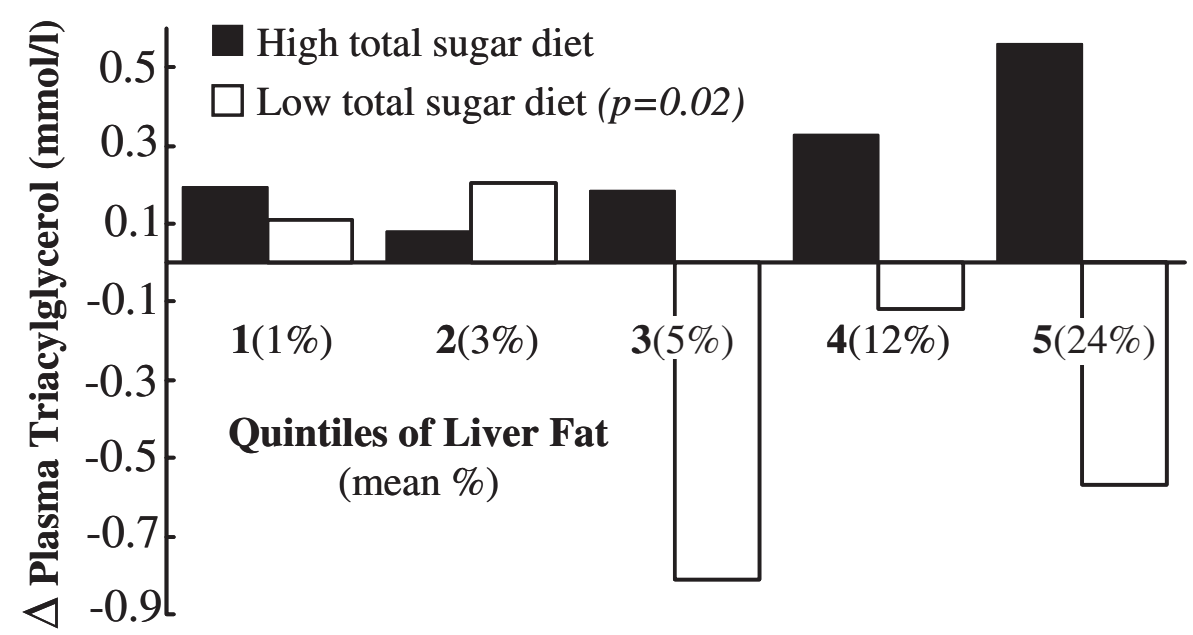

Across increasing quintiles of liver fat, plasma TAG generally increased and decreased on the high and low sugar diets, respectively. Subdivision of the cohort around the median of percentage liver fat $(4.2 \%)$, revealed a significant difference in plasma TAG between groups with moderately high $(n=12)$ and low liver fat $(n=13)$ after the high sugar diet $(P=0.02$, ANCOVA), but not after the low sugar diet. These data provide evidence to suggest that an elevated percentage of liver fat can influence the capacity of extrinsic sugars to increase plasma TAG, and further support the role of liver fat in mediating the potentially adverse effects of extrinsic sugars on plasma lipoproteins. This work was funded by the Biotechnology and Biological Sciences Research Council (Grant No. BB/G009899/1).

1. Stanhope KL \& Havel PJ (2008) Curr Opin Lipidol 19, 16-24.

2. Jebb SA, Lovegrove JA, Griffin BA et al. (2010) Am J Clin Nutr 92, 748-758.

3. National Diet \& Nutrition Survey: adults aged 19 to 64 years, volume 2 (2003) HMSO 